\title{
MedienPädagogik
}

Zeitschrift für Theorie und Praxis der Medienbildung

\section{KuDiKuPa - Kultur der Digitalität = Kultur der Partizipation?!}

\section{Verschränkung von Theorie und Praxis in partizipativ angelegter Hochschullehre durch Gaming und Game Design - ein Praxisbeispiel}

\author{
Daniel Autenrieth ${ }^{1}$ und Stefanie Nickel $^{2}$ (D) \\ ${ }^{1}$ Pädagogische Hochschule Ludwigsburg \\ ${ }^{2}$ Pädagogische Hochschule Schwäbisch Gmünd
}

\section{Zusammenfassung}

Der Beitrag gibt Einblick in ein dialogisch angelegtes, partizipativ organisiertes Hochschulseminar und rückt praxiserforschende Aspekte zwischen Schulen, Hochschulen und Bildungslandschaft auf Basis eines Modells in den Fokus. Über theoretische Ausgangspunkte wird der Frage nachgegangen, wie eine Kultur der Digitalität durch politisch-kulturelle Medienbildung zu einer Kultur der Partizipation werden kann. Weiterhin wird der Begriff der Partizipation betrachtet und danach gefragt, wie Gaming und Game Design einen Beitrag zu Partizipationserfahrungen leisten können. Zentral für die Seminargestaltung ist in dem aufgespannten theoretischen Rahmen ein Blick auf die Trias von partizipatorischer Schul- und Unterrichtsentwicklung. Den Abschluss des Beitrags bilden Praxiseinblicke in die im Rahmen eines Seminars erstellten digitalen Escape Games sowie den dahinterliegenden Entstehungsprozess. Dazu präsentiert der Beitrag Datenmaterial aus Forschungstagebüchern. 


\title{
Culture of Digitality = Culture of Participation?! Interweaving Theory and Practice in Participatory Teacher Education through Gaming and Game Design - A Practical Example
}

\begin{abstract}
The article provides an insight into a dialogically designed, participatively organized university seminar and focuses on practical research aspects between schools, universities, and the educational landscape. Theoretical starting points are used to explore the question of how a culture of digitality can become a culture of participation through political-cultural media education. Furthermore, the concept of participation will be considered and it will be asked how gaming and game design can contribute to experiences of participation. Central to the seminar design is a look at the triad of participatory school and classroom development within the theoretical framework. The article concludes with practical insights into the digital escape games created in the course of a seminar and the process behind their creation. In addition, the article presents data material from research diaries.
\end{abstract}

\section{Problemaufriss und Einführung}

«Handeln bezieht sich nicht auf die Intentionen, die Menschen beim Tun von Dingen haben, sondern auf ihr Vermögen, solche Dinge überhaupt zu tun (weshalb Handeln Macht impliziert, man vergleiche die Definition des Handelnden im Oxford English Dictionary als 〈jemand, der Macht ausübt oder eine Wirkung hervorruft〉). Handeln betrifft Ereignisse, bei denen ein Individuum Akteur in dem Sinne ist, dass es in jeder Phase einer gegebenen Verhaltenssequenz anders hätte handeln können. Was immer auch geschehen ist, es wäre nicht geschehen, wenn das Individuum nicht eingegriffen hätte» (Giddens $1988,60)$ 
Teilhabe an der Gestaltung von Schule und Unterricht sowie Gesellschaft setzt ein hohes Mass an Gestaltungswillen voraus. Um jedoch einen Gegenhorizont aufzubauen, zeigt die Erfahrung, dass sich Studierende häufig klar festgelegte Strukturen und Leitung wünschen. Der Wunsch nach vorgegebenen Wegen steht demzufolge pädagogischer Freiheit und kreativem Handeln sowie divergentem Denken im Weg. Mit anderen Worten: Das Bedürfnis zur Gestaltung von Welt und Zukunft steht in starkem Widerspruch zu unhinterfragten, tradierten Strukturen im Bildungsbereich, angefangen bei räumlichen und zeitlichen Strukturen, über curricular vorstrukturierte Abläufe bis hin zu Inhalten sowie Macht- und Hierarchie-Gefügen, in die die Studierenden aufgewachsen und eingebettet sind. In Anlehnung an das Habituskonzept von Bourdieu gehen wir davon aus, dass häufig nur Wahrnehmungen zugelassen werden, die im Einklang mit dem eigenen Verarbeitungsmodus stehen, um sich selbst vor krisenhaften Erfahrungen und sich daraus ergebenden Veränderungen zu schützen (Koller 2012, 27). Obwohl der Habitus, «der mit den Strukturen aus früheren Erfahrungen jederzeit neue Erfahrungen strukturieren kann» (Bourdieu 1987, 113f.), als etwas schwer Veränderliches gilt, macht Bourdieu deutlich, dass Erfahrungen auch umgekehrt die «alten Strukturen in den Grenzen ihres Selektionsvermögens beeinflussen» (ebd.).

Wir möchten mit den hier vorgestellten Ansätzen Strukturen aufbrechen und Möglichkeitsräume zum Ausprobieren, Fehler machen sowie kreativen und gestalterischen Handeln schaffen, die künftige Lehrkräfte dabei unterstützen, eine Kultur der Digitalität in einer Art und Weise mitzuprägen, die als Kultur der Partizipation verstanden und die Teilhabe und Gestaltung für alle ermöglichen kann. Unter Handeln verstehen wir daher in Anlehnung an Giddens (1988) einen kontinuierlichen Prozess der Alltagssteuerung, das heisst, Akteurinnen und Akteure sind Initiatorinnen und Initiatoren von Dingen, die er oder sie nicht beabsichtigt, aber dennoch hervorbringt. Handeln bezieht sich somit nicht auf die Intentionen, die Menschen beim Ausführen von Tätigkeiten haben, sondern auf die «Macht», Dinge zu tun und jederzeit auch anders handeln zu können (Giddens 1988, 60). 
Hochschuldidaktisch organisierte Lehr-Lernprozesse sind in Strukturen eingebettet. Seminare orientieren sich an Modulstrukturen und sind weitgehend vorstrukturiert. Das heisst, sie lassen in Bezug auf Inhalte wenig Spielraum für Mitgestaltung, Mitsprache und Mitbestimmung durch die Studierenden zu. Um dennoch Teilhabe zu ermöglichen, verfolgen wir das Prinzip einer dialogisch angelegten Praxisforschung zwischen Schulen, Hochschulen und der lokalen Bildungslandschaft und die damit einhergehende Verzahnung von Theorie und Praxis. Kooperationen zwischen den genannten Stakeholdern ermöglichen das Entwickeln und Erproben von neuen Ansätzen direkt im Unterricht im Sinne von: «von der Praxis für die Praxis〉. Synergieeffekte können genutzt werden, um auch einen gemeinsamen Austausch über Best Practice anzuregen. Mit einer Öffnung hin zu projektbezogenen und handlungsorientierten Formaten der Mitgestaltung geht einher, dass Studierende sich selbst Strukturen schaffen, eigenständig planen und strategisch vorgehen müssen. Rückmeldungen aus handlungsorientierten Hochschulseminaren zeigen, dass diese Freiheiten auf Zuspruch stossen (vgl. u. a. Niesyto 2019a) und subjektimmanente habituelle Strukturen verändert werden können, wenn genügend Zeit für gestalterisches Handeln im Rahmen von Seminaren zur Verfügung gestellt wird und der entsprechend höhere Workload auch angerechnet werden kann (ebd. 2019a, 227). Eine Teilnehmerin des Seminars, welches weiter unten noch genauer skizziert wird, beschrieb diese Art des Arbeitens als

«[...] Lernen ausserhalb der Komfortzone. Die eigenständige Erstellung eines Escape-Rooms war ein Novum für mich, das einen Zugewinn an Lebenskompetenz bzw. Nützliches für späteres professionelles und privates Fortkommen [...] darstellt» (interne Seminarevaluation).

Damit ist der Problemhorizont eröffnet: Wir werden einen Einblick in ein Hochschulseminar geben, welches dialogisch angelegte, partizipativ organisierte Praxisforschung zwischen Schulen, Hochschulen und Bildungslandschaft in den Fokus rückt. Die Basis hierfür bieten theoretische Ausgangspunkte, in denen wir der Frage nachgehen, wie eine Kultur der Digitalität durch eine politisch-kulturelle Medienbildung zu einer Kultur der Partizipation werden kann. Weiterhin betrachten wir den Begriff der 
Partizipation und fragen danach, wie Gaming und Game Design einen Beitrag zu Partizipationserfahrungen leisten können. Zentral für die Seminargestaltung ist ein Blick auf die Trias von partizipatorischer Schul- und Unterrichtsentwicklung. Den Abschluss des Beitrags bilden Einblicke, in die im Rahmen des Seminars erstellten digitalen Escape Games sowie den dahinterliegenden Entstehungsprozess.

\section{Theoretischer Rahmen}

\subsection{Kultur der Digitalität = Kultur der Partizipation?}

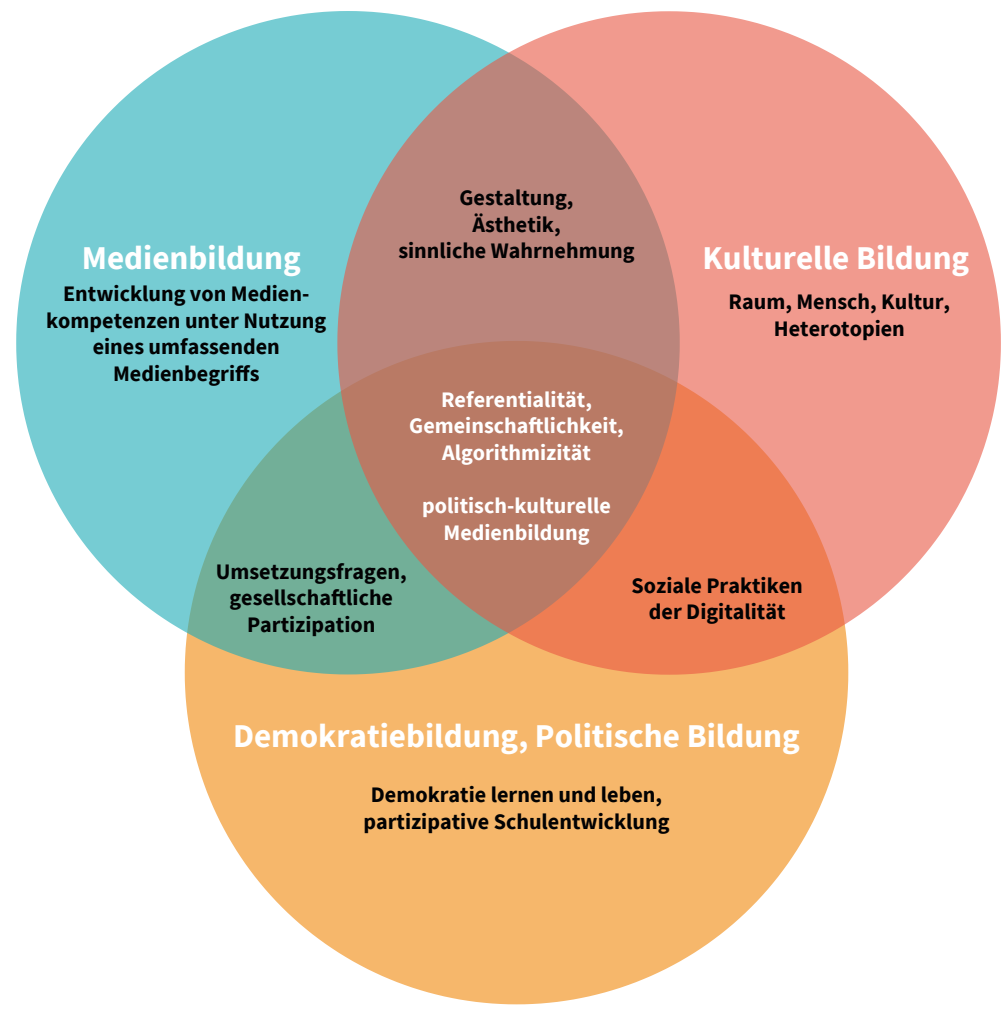

Abb. 1: Modell einer politisch-kulturellen Medienbildung (eigene Darstellung). 
Um ein kritisch-reflektiertes Verständnis sowie Handlungsoptionen innerhalb einer Kultur der Digitalität zu entwickeln, bedarf es eines interdisziplinären Zugangs zur Betrachtung der Welt und der vorherrschenden Kultur (Autenrieth, Baumbusch, und Marquardt 2020). Das in Abbildung 1 dargestellte Modell unterstreicht, dass die digital vernetzte Welt sowie die daraus resultierenden Phänomene, Gegenstände und Situationen als Teil des Metaprozesses (Krotz 2007, 11) Digitalisierung weder räumlich noch zeitlich in sozialen und kulturellen Folgen begrenzt sind. Digitalisierung ist zugleich auch ein Produkt der menschlichen Kultur (Rat für Kulturelle Bildung 2019) und hat somit Auswirkungen darauf, wie Menschen leben, welche Wahrnehmungsstrukturen sie ausbilden und nutzen und wie sie ihre Umwelt gestalten. Letztlich ergeben sich aus der Digitalisierung im Kontext ökonomischer und politischer Interessen aber auch Problemfelder, etwa bei der Verwertung persönlicher Daten oder der Herausbildung totalitärer und kommerzieller Machtstrukturen, die ausserhalb einer gesellschaftlichen Kontrolle liegen (Niesyto 2019b). In einer Kultur der Digitalität, die nach Stalder durch die Eigenschaften Referenzialität, Gemeinschaftlichkeit und Algorithmizität (Stalder 2019, 13) geprägt ist, trägt der Umgang mit Formen von Kultur zur Veränderung der Gesellschaft bei. Dichotome Möglichkeiten wären: eine post-demokratische Welt der Überwachung, die geprägt ist durch Wissensmonopole, oder eine «Kultur der Commons und der Partizipation» (Stalder 2019). Beide Pole sollen im Folgenden beispielhaft skizziert werden, um im Anschluss die Bedeutung einer politisch-kulturellen Medienbildung herauszustellen.

Die Entwicklung der Corona Warn App in Deutschland im Jahr 2020 hat gezeigt, wie stark beispielsweise das Kräfteungleichgewicht zwischen demokratisch legitimierten Regierungen und Unternehmen sein kann. Die Bundesrepublik wollte gegenüber Apple und Google erreichen, dass ihre Schnittstelle auch eine zentrale Speicherlösung von Begegnungsdaten ermöglicht (Krempl 2020). Dass sich im Falle der Corona-Warn-App nun ein dezentraler Speicheransatz durchsetzte, wie ihn viele Datenschützende wünschten (Apple und Google hatten ebenfalls darauf gedrängt), ist aber durchaus nachvollziehbar. Denn eine dezentrale Lösung ist auch für die beteiligten Unternehmen bequemer und sicherer. Dass sich Staaten den beiden Unternehmen anpassen mussten, zeigt aber das angesprochene 
Kräfteungleichgewicht. Die Macht hatten in diesem Fall diejenigen, die auch die Macht über die Infrastruktur haben. Zu betonen ist weiter, dass die grundlegenden technischen Voraussetzungen der Corona App von Google und Apple bereitgestellt werden. Dies geschah nicht nur im Bereitstellen einer Plattform wie iOS oder Android, sondern auch durch die entsprechende Entwicklung von Schnittstellen zur Kontakterkennungen über die Bluetooth Funktechnik im Hintergrund. Ulrich Dolata (Techniksoziologe) kam nach einer Untersuchung zu Märkten und Macht der Internetkonzerne bereits im Jahr 2014 zu dem Schluss, dass die damals untersuchten fünf Konzerne (Google, Facebook, Apple, Amazon und Microsoft) nicht nur wesentliche Angebote und Märkte des Internets prägten. Vielmehr regeln sie als Betreiber der zentralen Infrastrukturen auch die Art und Weise, wie Menschen Zugang zum Internet erhalten und wie der Zugang zu Informationen und zu Kommunikation darin strukturiert wird (Dolata 2014). Dolata unterstreicht, dass

«[n]icht Dezentralisierung, Demokratisierung und Kooperation, sondern Konzentration, Kontrolle und Macht [...] die Schlüsselprozesse und -kategorien [sind], mit denen sich die wesentlichen Entwicklungstendenzen des (kommerziellen) Internets angemessen erfassen lassen.» (ebd., 5f.)

Im Gegensatz dazu stehen die von Stalder beschriebenen Commons. Er unterscheidet diese in drei Dimensionen, nämlich Güter, die gemeinschaftlich genutzt werden, Mitglieder von Gemeinschaften, die die Ressource herstellen, pflegen und nutzen sowie Praktiken, Normen und Institutionen, die von den Gemeinschaften selbst entwickelt werden (Stalder 2019, 246).

Die Mitglieder der Gemeinschaft, die Commoners sind «in unterschiedlichem und variablem Ausmass Produzenten und Konsumenten der gemeinsamen Ressourcen» (ebd., 247) und haben so Teil an der Gesamtdynamik der Commons. Mit Blick auf gesellschaftliche und medienbezogene Herausforderungen betonen Aufenanger et al., dass Partizipation im öffentlichen Bereich und an politischen Entscheidungsprozessen aber auch bei der aktiven Gestaltung eigener Lernprozesse in einer von der 
Digitalität geprägten Welt zentrale Themen sind (Aufenanger, Eickelmann, und Herzig 2014, 34). Open Source Software, Open Data oder Open Educational Resources sind nur drei Beispiele einer solchen Kultur der Commons.

\section{Politisch-kulturelle Medienbildung}

Die skizzierten Beispiele verdeutlichen den offenen Ausgang der weiteren Entwicklung der Digitalisierung und den Bedarf an einer aktiven Mitgestaltung des Prozesses. Anhand der Verschiedenartigkeit der dargestellten Eigenschaften zeigt sich auch der Bedarf an einer interdisziplinären Betrachtung einer Kultur der Digitalität. Unterschiedliche Disziplinen der Pädagogik können hier zusammenwirken (siehe Abb. 1). So ist eine umfassende Medienbildung unerlässlich für gesellschaftliche Teilhabe, für die Partizipation im öffentlichen Bereich und an politischen und demokratischen Entscheidungsprozessen. Demokratie ist das Ergebnis menschlichen Handelns und menschlicher Erziehung und muss erlernt werden. Kultur umfasst Prozesse, «in denen soziale Bedeutung, also die normative Dimension der Existenz, [...] verhandelt oder realisiert [wird]» (Stalder 2019, 16). Die Kulturelle Bildung eröffnet (auch ästhetisch-kreative) Zugänge und Erfahrungsräume im Rahmen dieser Prozesse. Demokratiebildung, Kulturelle Bildung und Medienbildung schaffen durch ihre wechselseitigen Beziehungen Ergänzungspotenziale sowie Schnittmengen von Zielen, Methoden und Betrachtungsgegenständen Möglichkeiten zur gegenseitigen Befruchtung (Autenrieth und Nickel 2021; 2020). Sie können gemeinsam im Rahmen von Bildungsprozessen entlang der gesamten Bildungskette dazu beitragen, dass die Kultur der Digitalität sich auch zu einer freiheitlichen Kultur der Partizipation entwickelt. Soll Bildung in diese Richtung entwickelt werden, müssen Bildungsorte mit entsprechenden Konzepten Menschen dazu befähigen, souverän, mündig und gestaltend an der Gesellschaft teilzuhaben. Das funktioniert nur, wenn Menschen die Möglichkeit erhalten, ihre Fähigkeiten und Fertigkeiten freizusetzen, anzuwenden und zu lernen, im 21. Jahrhundert handlungsfähig zu sein. Dazu zählen unter anderem folgende Aspekte (Trilling und Fadel 2009):

- sich zu informieren,

- Wissen eigenständig zu erwerben und zu teilen,

- grenzübergreifend zu kommunizieren, 
- mit Veränderungen und Komplexität umzugehen,

- kreative Lösungen zu finden oder

- kollaborativ, interdisziplinär und multiperspektivisch zu arbeiten.

Mündige Bürgerinnen und Bürger können - mit und durch Medien aktiv gestalten bzw. verändern und reproduzieren nicht einfach nur unhinterfragte strukturelle Gegebenheiten. Dies schärft die Bedeutung, in Schule und Lehrkräftebildung Möglichkeiten zur Partizipation in den Lebenswelten von Kindern, Jugendlichen und jungen Erwachsenen zu schaffen und damit Beteiligung und Selbstbestimmung erfahrbar zu machen.

\subsection{Der Partizipationsbegriff}

Partizipation, Teilhabe und Engagement sind Begriffe, die häufig im Kontext politischer Prozesse verwendet werden. Wir möchten allerdings von einem weiteren Verständnis ausgehen, das sowohl die Eigenschaften von Beteiligungsformen in einer post-digitalen ${ }^{1}$ Welt miteinbezieht als auch gerade im Kontext der kulturellen Bildung eine zentrale Bedeutung für die Interaktion zwischen Institutionen und Subjekten darstellt.

Mit Blick auf eine Kultur der Digitalität arbeitete Soßdorf heraus, «dass Partizipation im Netz nicht einzig als Übertragung 〈klassischer Beteiligungsformen ins Virtuelle) (Wimmer 2012a, S. 31) betrachtet werden kann. Diesen adaptierten Partizipationsangeboten unterstellt [Wimmer] eine Top-Down-Orientierung, bei denen dem Nutzer keine ausreichende Mitbestimmung und Transparenz geboten werden. Doch gerade die von Nutzern selbst entwickelten und initiierten Aktionen, die als Graswurzelbewegungen stattfinden und die Grenzen zwischen online und offline verwischen, beschreibt er als typische, neue Formen einer digitalen Deliberation und Partizipation (Wimmer 2012a, S. 27 ff.; vgl. Best/Krueger 2005).» (Soßdorf $2016,41)$

1 Der Ausdruck post-digital deutet hier nicht auf das Ende des Digitalen hin, sondern auf das Ende der Auffassung des Digitalen als spezifisches kulturelles Differenzkriterium gegenüber einer nicht-digitalen Weise des Seins. Anders ausgedrückt: Es geht um die gefühlte Selbstverständlichkeit und die Omnipräsenz des Digitalen, die in der Lebensrealität nur noch bei Abwesenheit oder Fehlfunktionen bemerkt wird (u. a. Schmidt 2020). 
Von Nutzerinnen und Nutzern initiierte Aktionen und Eigenproduktionen spricht auch Simon eine grosse Bedeutung zu. Besonders kulturelle Institutionen (diese begreifen wir als Teil lokaler Bildungslandschaften, siehe dazu auch den Abschnitt 2.3) können diese Art von Prozessen ermöglichen, indem sie als Plattformen agieren, die Menschen zusammenbringen und ihnen ermöglichen, zu Erstellerinnen und Erstellern und Botschafterinnen und Botschaftern für Inhalte sowie zu Kritikerinnen und Kritikern und damit zu Mitgestaltenden zu werden (Simon 2010, 2).

Zur Einordnung von Partizipationsmöglichkeiten eignet sich das Partizipationsverständnis des amerikanischen Psychologen Roger Hart. Es beruht auf seiner Ansicht, dass Partizipation ein Prozess der Entscheidungsfindung ist, welcher das eigene Leben und das Leben einer Gemeinschaft beeinflusst, in der das Individuum lebt.

«A nation is democratic to the extent that its citizens are involved, particularly at the community level. The confidence and competence to be involved must be gradually acquired through practice. It is for this reason that there should be gradually increasing opportunities for children to participate in any aspiring democracy, and particularly in those nations already convinced that they are democratic» (Hart 1992, 4).

In der demokratiepädagogischen Diskussion um Partizipation findet sich eine Reihe von Stufenmodellen zur Beteiligung von Kindern und Jugendlichen bzw. Schülerinnen und Schülern (Nickel 2016; Hart 1992) im institutionellen Kontext. Die operationalisierten Stufenmodelle definieren Ränge, die eine Untersuchung der Handlungsformen in der Praxis ermöglichen. Auch Hart formuliert ein solches Stufenmodell, an dem wir uns im Weiteren orientieren werden: 


\begin{tabular}{|c|c|c|}
\hline \multirow{5}{*}{ 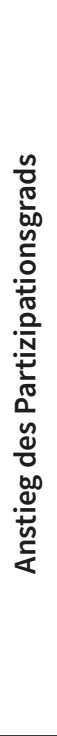 } & $\begin{array}{l}\text { Stufe } 8 \\
\text { child-initiated, sha- } \\
\text { red decisions with } \\
\text { adults }\end{array}$ & $\begin{array}{l}\text { Kinder/Schülerinnen und Schüler können eigene } \\
\text { Projekte vorschlagen und Erwachsene/Schule (Schul- } \\
\text { leitung/Lehrpersonen) helfen bei der Durchführung } \\
\text { und Umsetzung. }\end{array}$ \\
\hline & $\begin{array}{l}\text { Stufe } 7 \\
\text { child initiated and } \\
\text { directed }\end{array}$ & $\begin{array}{l}\text { Kinder/Schülerinnen und Schüler können eigene Pro- } \\
\text { jekte vorschlagen und selbstständig durchführen. }\end{array}$ \\
\hline & $\begin{array}{l}\text { Stufe } 6 \\
\text { adult-initiated, sha- } \\
\text { red decisions with } \\
\text { children }\end{array}$ & $\begin{array}{l}\text { Entscheidungen und Projekte werden von Erwachse- } \\
\text { nen/der Schule initiiert, die Kinder/Schülerinnen und } \\
\text { Schüler werden informiert und in Abstimmungspro- } \\
\text { zesse mit einbezogen. }\end{array}$ \\
\hline & $\begin{array}{l}\text { Stufe } 5 \\
\text { consulted and } \\
\text { informed }\end{array}$ & $\begin{array}{l}\text { Entscheidungen werden von Erwachsenen/der } \\
\text { Schule getroffen, Kinder/Schülerinnen und Schüler } \\
\text { werden informiert und haben beratende Funktion. }\end{array}$ \\
\hline & $\begin{array}{l}\text { Stufe } 4 \\
\text { assigned but infor- } \\
\text { med }\end{array}$ & $\begin{array}{l}\text { Kindern/Schülerinnen und Schülern wird eine Rolle } \\
\text { zugewiesen, sie werden aber ausreichend über } \\
\text { Prozesse und Inhalte der getroffenen Entscheidungen } \\
\text { informiert. }\end{array}$ \\
\hline \multirow{3}{*}{ 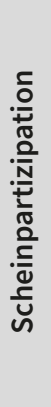 } & $\begin{array}{l}\text { Stufe } 3 \\
\text { tokenism }\end{array}$ & $\begin{array}{l}\text { Kinder/Schülerinnen und Schüler werden bei wich- } \\
\text { tigen Entscheidungen gefragt, haben aber keine } \\
\text { Möglichkeit eine eigene Meinung zu bilden bzw. eine } \\
\text { Wahl zu treffen. }\end{array}$ \\
\hline & $\begin{array}{l}\text { Stufe } 2 \\
\text { decoration }\end{array}$ & $\begin{array}{l}\text { Kinder/Schülerinnen und Schüler werden über wich- } \\
\text { tige Entscheidungen und Projekte informiert, dürfen } \\
\text { aber nicht mitbestimmen. }\end{array}$ \\
\hline & $\begin{array}{l}\text { Stufe } 1 \\
\text { manipulation }\end{array}$ & $\begin{array}{l}\text { Wichtige Entscheidungen und Projekte werden ohne } \\
\text { Mitwirkung der Kinder/Schülerinnen und Schüler } \\
\text { getroffen und durchgeführt. }\end{array}$ \\
\hline
\end{tabular}

Tab. 1: From Tokenism to Citizenship (eigene Darstellung in Anlehnung an Hart 1992).

Mit Blick auf die «Ladder of Participation» von Hart stellt sich uns im Kontext der Lehrkräfteausbildung die Frage, wie im Rahmen von Seminarangeboten innerhalb vorgegebener Modulstrukturen ein möglichst hoher Partizipationsgrad erreicht werden kann. Die bereits im Problemaufriss angesprochene dialogisch angelegte und projektbasierte Praxisforschung bietet Freiräume für Studierende, eigene (Teil)Projekte zu verwirklichen und damit reale Partizipationserfahrungen zu sammeln. Lernprozesse laufen in diesem Kontext nicht linear, fremdgesteuert oder vorhersagbar ab, sondern vor allem nicht linear und selbstorganisiert. Gleichzeitig erleben 
und reflektieren die Studierenden eine Veränderung der Professionalität von Lehrenden. Es geht nicht mehr um das Lehren, Vermitteln und Führen, sondern um das Begleiten, Beraten und Unterstützen und damit letztlich um die Entwicklung und Konstruktion reflexiven Wissens. Unterricht kann dann verstanden werden als Realisierung und Begleitung von Lernprojekten und die Gültigkeit der Wirklichkeitskonstruktion wird im Dialog reflektiert und problematisiert. Eine Individualisierung und Pluralisierung wird möglich (Arnold und Schön 2019, 54).

Aus diesen - möglichst zufriedenstellenden - Partizipationserfahrungen heraus können die angehenden Lehrkräfte ihr eigenes Professionsverständnis weiterentwickeln und so in ihrer späteren beruflichen Praxis an den Lebenswelten von Schülerinnen und Schülern orientierte, reale Teilhabemöglichkeiten schaffen.

\subsubsection{Game design und Partizipation}

«Im Spiel werden die bekannten Strukturen und Ordnungen des Lebens porös. Im Spiel tauchen wir ein in jene Potenziale, die zu entfalten uns lebendig macht. Im Spiel eröffnen sich uns neue Perspektiven.» (Hüther und Quarch 2016, 72)

Dieses Zitat unterstreicht die Bedeutung des Spiels als Grundlage menschlicher Kultur (Huizinga 1956, 51) und hat damit grossen Einfluss auf und Bedeutung für menschliches Verhalten. Besonders digitale Spielwelten sind für viele Menschen heute prägend und Teil ihrer Lebenswelt. Nicht nur 68\% der Kinder und Jugendlichen spielen regelmässig digital (Feierabend, Rathgeb, und Reutter 2020, 53). Bereits 2004 beschrieben Beck und Wade, wie die Kohorte der «Gamer Generation» (geboren ab ca. 1975) die Baby Boomer zahlenmässig überholt (Beck und Wade 2004, 17). Eine Untersuchung der Entertainment Software Association aus dem Jahr 2020 beschreibt die durchschnittliche Altersspanne von Gamerinnen und Gamern in den USA zwischen 35 und 44 Jahren. Weiterhin spielen ebenfalls 64\% aller Erwachsenen in den USA (163,3 Millionen Menschen) regelmässig digitale Spiele. Digitale Spiele sind daher aus zweierlei Hinsichten mit Blick auf Partizipation hoch relevant. 


\subsubsection{Lebensweltbezug}

Im Rückgriff auf Husserl ist die Lebenswelt die selbstverständliche Basis des alltäglichen Denkens und Handelns (Husserl 1996). Die oben skizzierten Zahlen verdeutlichen, dass für einen immer grösser werdenden Teil der Gesellschaft vielschichtige Spielerlebnisse im virtuellen Raum zur alltäglichen Lebenswelt gehören. Obwohl Spielhandlungen keine direkten Konsequenzen in der realen Welt nach sich ziehen, haben sie dennoch Bedeutung für die Wirklichkeit. Beranek und Ring (2016) gehen daher davon aus, dass Digitale Spielwelten aufgrund ihrer sozialen Strukturen, ihrer Lebensweltnähe und der Ansprache intrinsischer Motivation als Räume für Partizipationserfahrungen dienen können. In Anlehnung an die Partizipationsleiter (Hart 1992) und die Partizipationsstufen der politischen Offline- und Online-Partizipation (Soßdorf 2016, 174) haben Beranek und Ring (2016) eine Tabelle mit den Stufen der Beteiligung in digitalen Spielwelten entwickelt:

\begin{tabular}{|c|c|c|}
\hline & Stufen & Aktivitäten der Spielenden \\
\hline \multirow{2}{*}{ 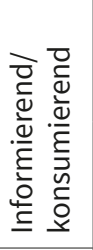 } & $\begin{array}{l}\text { Stufe } 1 \\
\text { Information/ } \\
\text { Konsum }\end{array}$ & $\begin{array}{l}\text { z. B. sich über Spiele informieren, Spiel kaufen, Spiel } \\
\text { spielen }\end{array}$ \\
\hline & $\begin{array}{l}\text { Stufe } 2 \\
\text { Positionierung/ } \\
\text { Zuordnung }\end{array}$ & $\begin{array}{l}\text { Sich auf ein Spiel einlassen, Involvement, sich als } \\
\text { Gamerin/Gamer «outen», Werbematerial kaufen }\end{array}$ \\
\hline \multirow{2}{*}{ 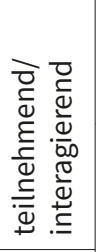 } & $\begin{array}{l}\text { Stufe } 3 \\
\text { Teilnahme }\end{array}$ & $\begin{array}{l}\text { Soziale Beziehungen eingehen (z. B. Freundschaften } \\
\text { eingehen, Beteiligung z. B. durch Beteiligung auf } \\
\text { YouTube: liken, Kommentar, Diskussion) }\end{array}$ \\
\hline & $\begin{array}{l}\text { Stufe } 4 \\
\text { Sich einbringen }\end{array}$ & $\begin{array}{l}\text { In der Gemeinschaft aktiv werden (in einem Clan } \\
\text { aktiv werden; Herstellersupport kontaktieren; Ideen } \\
\text { einbringen) }\end{array}$ \\
\hline \multirow{2}{*}{ 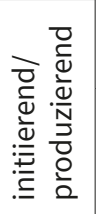 } & $\begin{array}{l}\text { Stufe } 5 \\
\text { Aktion }\end{array}$ & $\begin{array}{l}\text { Eigene Videos z. B. Let's Play veröffentlichen oder } \\
\text { auch Aktionen ausserhalb des Spiels starten }\end{array}$ \\
\hline & $\begin{array}{l}\text { Stufe } 6 \\
\text { Modifikation/ } \\
\text { Produktion }\end{array}$ & $\begin{array}{l}\text { Hacking, Modding } \\
\text { Eigene Spiele designen } \\
\text { Gameserver aufbauen }\end{array}$ \\
\hline
\end{tabular}

Tab. 2: Stufen der Beteiligung in digitalen Spielwelten (vereinfachte Darstellung nach Beranek und Ring 2016). 
Durch das grosse Angebot niedrigschwelliger und teilweise sogar kostenloser Software ist es möglich, auch mit technisch unerfahrenen Studierenden, Schülerinnen und Schülern, Spielideen nicht nur zu entwickeln, sondern diese auch umzusetzen. Die Bandbreite reicht von Textadventures bis hin zu 3D Games. So ergeben sich durch die Produktion eigener Spielwelten erfolgreiche Partizipationserfahrungen, die geprägt sind durch ein hohes Mass an Selbststeuerung sowie eigenständigem, autonomem Handeln. Die Entwicklung von Spielen geht einher mit Aspekten des Storytellings, Audio- und Videoproduktionen sowie der Bildbearbeitung. Es findet also auch ein Lernen mit und über Medien statt, was die Anbahnung von Medienkompetenz und durch die Reflexion dieser Prozesse auch medienpädagogische Kompetenzen unterstützt. Diese Art des handlungsorientierten Arbeitens eignet sich in Schule, Hochschule und in Kooperation mit der Bildungslandschaft für Kleingruppenarbeit und die Aneignung der wichtigen Zukunftskompetenzen Kommunikation, Kollaboration, Kreativität und kritisches Denken. Mit Blick auf das Ziel der Gestaltung von Welt schreibt u. a. Theunert der kommunikativen Kompetenz eine besondere Bedeutung zu:

«[Sie] steht mithin für die Fähigkeit zu selbstbestimmter, reflexivkritischer Kommunikation und bildet die Grundlage für Aneignung von, aktives Einwirken auf und Veränderung von Realität» (Theunert 2009, 200).

\subsubsection{Eigenschaften von Spielen}

Spieleentwicklerinnen und -entwickler entwickeln Settings, die Spielerinnen und Spieler zu extremen Anstrengungen inspirieren und harte Arbeit wertschätzen. Sie regen Menschen zu Kooperation und Kollaboration an. Sie schaffen es, Spielerinnen und Spieler zu motivieren, immer schwieriger und komplexer werdende Aufgaben zu meistern.

«These crucial twenty-first-century skills can help all of us find new ways to make a deep and lasting impact on the world around us. Game design isn't just a technological craft. It's a twenty-first-century way of thinking and leading. And gameplay isn't just a pastime. It's a twenty-first-century way of working together to accomplish real change.» (McGonigal 2012, 13) 
Die Spieleforscherin McGonigal skizziert hier das grosse Potenzial, das einhergeht mit der immer grösser werdenden «Gamer Generation» und erörtert, warum alle Menschen von Computerspielen profitieren und wie sie die Welt verändern können. Ausgangspunkt ihrer Überlegungen ist ihr Urteil: «Reality, compared to games, is broken» (McGonigal 2012, 3). Sie nennt dafür unter anderem folgende Gründe (ebd.):

- Die Realität motiviert Menschen nicht in dem Masse, wie es Computerspiele können.

- Die Realität ist nicht in einer Art und Weise arrangiert, dass sie das grösstmögliche Potenzial jedes Einzelnen entfaltet.

- Die Realität wurde nicht gestaltet, um Menschen glücklich zu machen.

Im Problemaufriss hatten wir beschrieben, dass Teilhabe an der Gestaltung von Schule und Unterricht sowie Gesellschaft ein hohes Mass an Motivation zur Gestaltung voraussetzt. Motivation (insbesondere intrinsische Motivation) als Motor menschlichen Handelns basiert auf Autonomie, Kompetenz und sozialer Eingebundenheit (Deci und Ryan 1993). All diese Merkmale erfüllen erfolgreiche Spiele. Csíkszentmihályi fügt diesen Merkmalen noch das «Flow»-Erlebnis hinzu, welches sich auszeichnet als ein Zustand zwischen Unterforderung (Bore-out) und Überforderung (Burn-out). In diesem hoch kreativen Zustand vergisst man Raum und Zeit, ist hochkonzentriert, lösungsorientiert, fokussiert und prozessorientiert (Csikszentmihalyi 2015).

Aus diesen Gründen nehmen wir an, dass Gaming und Game Design Menschen zu kreativem und kommunikativem Handeln motivieren, mit dem Ziel, aus den (digitalen oder analogen) Spielwelten Autonomie, Motivation und Kreativität zur Gestaltung von Welt zu schöpfen. Folgende Fragen sind daher für uns bei der Gestaltung von Lehr- und Lernprozessen zentral:

- Eignen sich Gaming und Game Design dafür, dass Menschen zu kreativem und kommunikativem Handeln angeregt werden?

- Welche Spiele und Spielformen eignen sich und warum?

Die nachfolgend dargestellte Seminargestaltung aus dem Master Bildungswissenschaften greift die genannten Aspekte auf und skizziert sie beispielhaft. 


\subsection{Seminargestaltung anhand der Trias von partizipatorischer Schul- und Unterrichtsentwicklung}

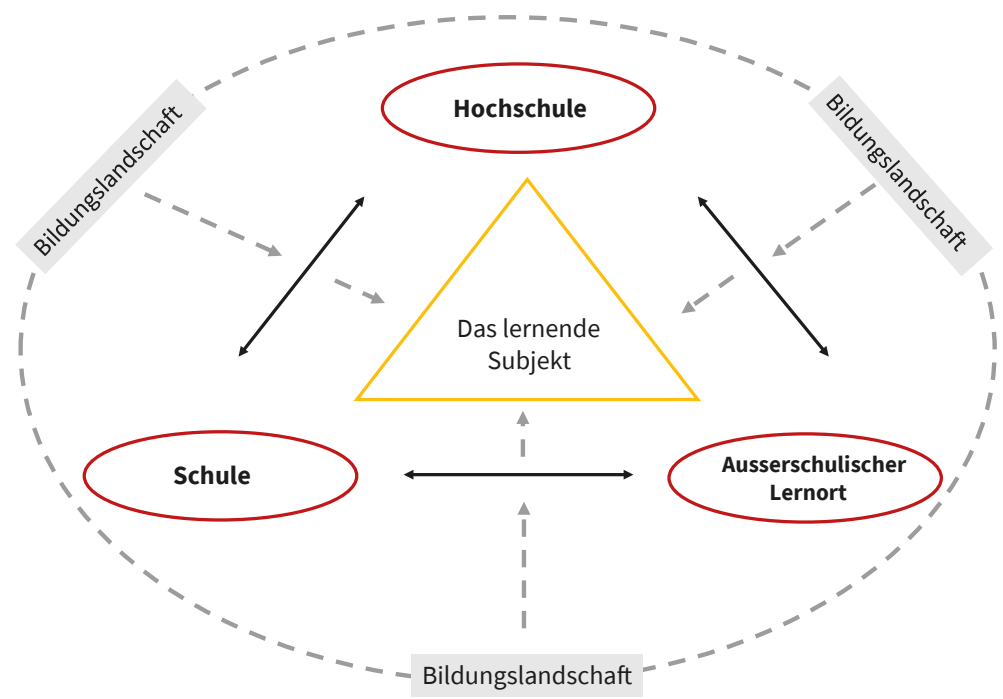

Abb. 2: Trias der partizipatorisch angelegten Schul- und Unterrichtsforschung eigene Darstellung in Anlehnung an Rolff 2016.

Im Mittelpunkt der Planung von Lehrveranstaltungen an der Hochschule stehen Studierende als mündige Subjekte des eigenen Lernprozesses (Abb. 2). Um auf die heterogene Zusammensetzung des Plenums einzugehen, ist eine differenzierte inhaltliche, didaktische und methodische Gestaltung notwendig, in der wechselnde Lehr-Lernformen eingesetzt werden. Insbesondere Kooperationen mit dem Ziel des Austauschs und der Einbettung in gegebene Strukturen nehmen eine entscheidende Rolle ein, weil sich Studierende mit Spezifika ihres Lernstandorts auseinandersetzen und identifizieren können. Hier bieten Kooperationen mit Schulen und Ausserschulischen Lernorten hervorragende Möglichkeiten. Das heisst, die Studierenden bearbeiten direkt an der Schnittstelle zwischen Universität und Gesellschaft theoretische Modelle, Konzeptionen und Positionen sowie empirische Forschungsergebnisse und praxisorientierte Beispiele, um darauf aufbauend Implikationen für die (eigene) professionelle Praxis abzuleiten. Besonderes Augenmerk sollte dabei auf die Ressourcen Raum und Zeit gelegt werden, um den Studierenden die Möglichkeit zu geben, gemeinsam 
vorhandenes Wissen und Neues zu reflektieren, Fragen zu stellen und sich miteinander auszutauschen, sodass Gestaltungsmöglichkeiten erkannt werden können. Unsere Annahme ist daher: Lernarrangements im Rahmen einer Verschränkung von Hochschule, Schule und ausserschulischem Lernort ermöglichen die Entfaltung der Persönlichkeit des Subjekts sowie die Anbahnung und den Aufbau pädagogischer Handlungspraxis.

In diesem Zusammenhang zeigt sich jedoch als eine generelle Herausforderung in der Hochschullehre die häufig von «Studierenden beklagte Praxisferne und Theorielastigkeit im Lehramtsstudium. Wir sind zu verkopft», ist ein Satz, der nicht selten von Studierenden in Seminaren geäussert wird. Ferner ist zu berücksichtigen, dass Studierende häufig in Seminar-Kontexten auf ein Verständnis von Schule aus der eigenen Schulzeit und damit verbundene Lehr- und Lern-Prozesse zurückgreifen. Damit einhergehende explizite und implizite Denkstrukturen können handlungsbestimmend sein. Als zentral für die hochschuldidaktische Planung von Lehr- und Lernprozessen erscheint daher die Verzahnung von Theorie und Praxis im Lehramtsstudium. Folglich gilt es, die Potenziale und Möglichkeiten des Hochschulstandortes zu berücksichtigen, um einen stärkeren Praxisbezug zu ermöglichen, durch beispielsweise projektorientierte Seminare gemeinsam mit Schulen und Bildungseinrichtungen vor Ort. So ist der auf Gegenseitigkeit beruhende Lehr- und Lernprozess auf das Schaffen eines kommunikativen und kooperativen Lernraums angewiesen, der wiederum bestimmten strukturellen Implikationen ausgesetzt ist: Studierende wünschen sich häufig konkrete Handlungskonzepte mit Blick auf die praktischen Phasen. Pädagogische Professionalität unterliegt jedoch organisationslogischen Strukturbedingungen bzw. Antinomien im pädagogischen Handeln (Helsper 2012; Oevermann 1981) sowie Unsicherheiten bzw. Ungewissheiten (Luhmann 1981), die sich aus der pädagogischen Alltagspraxis ergeben. Festgelegte wiederholbare Handlungskonzepte - im Sinne einer alltäglichen Best Practice-Umsetzung - kann es jedoch nicht geben. Pädagogische Professionalität baut vielmehr auf theoretischen, erfahrungspraktischen und reflektierten Wissensbeständen auf, mittels derer ein Repertoire für situatives Handeln und Gestalten angelegt werden kann. Im Sinne Luhmanns (1981) agieren professionelle Praktikerinnen und Praktiker im Bildungssystem als Vermittlerinnen und Vermittler 
zwischen einer Dichotomie, zum Beispiel zwischen Theorie und Praxis. Hier bietet sich die Einbindung von Projekten als Entwicklungsperspektive an, um eine Verschränkung beider Pole anzustossen. Kooperativ organisierte Formate ermöglichen den Lernenden, zu kreativ forschenden Gestaltenden zu werden und machen Lehrende zu Begleitenden.

Zusammenfassend kann festgehalten werden: In den dargelegten Gedanken zur Trias der partizipatorischen Schul- und Unterrichtsentwicklung spiegelt sich ein konstruktivistisch (Reich 2002) angelegtes, forschend-entdeckendes Lehr-Lernverständnis. Mit Blick auf die Verschränkung von Theorie und Praxis im Rahmen der Gestaltung von Lehr- und Lernprozessen an der Hochschule als standortbezogener Lehr- und Lernraum bedeutet dies:

1. Inhaltlich setzen sich die Studierenden mit theoretischen Grundlagen sowie technischen Aspekten auseinander und betten ausgewählte Lerngegenstände in aktuelle Diskurse ein, um Chancen und Herausforderungen von partizipatorisch orientierter Schul- und Unterrichtsentwicklung unter besonderer Berücksichtigung von Medien- und Demokratiebildung und kultureller Bildung im universitären und schulischen Kontext zu begegnen.

2. Aus didaktischer Perspektive erhalten Studierende die Möglichkeit, Lerngegenstände aus dem unmittelbaren Lebensumfeld aufzugreifen und inhaltlich an die Erforschung, Wahrnehmung und Erkundung im Raum anzubinden.

3. Wissenschaftlich-reflexiv und institutionell nachhaltig verorten lassen sich die Projekte und Seminare daher als Erkenntnisgewinnung aus dialogisch angelegter, praxiserforschender Perspektive durch eine Theorie-Praxis-Verzahnung im Lehramtsstudium.

Die beschriebenen theoretischen Begriffe, Annahmen und Aspekte finden Berücksichtigung in unserem Praxisbeispiel, welches wir nachfolgend skizzieren möchten. 


\section{Einblicke in die Praxis}

Beim Einsatz digitaler Medien in der Schule geht es um ein Lernen mit und über Medien, damit sich Schülerinnnen und Schüler selbstbestimmt und kritisch-reflexiv in ihren vielfältig geprägten Lebenswelten bewegen, sich selbstständig Urteile bilden und ihre kulturell-poltische Identität sowie Umwelt eigenständig erschliessen können. Im Zentrum einer an demokratischen Werten orientierten Schul- und Unterrichtsentwicklung steht das handelnde Subjekt mit seinen sozialen Praktiken. Bei der Thematisierung des Einsatzes digitaler Medien in Schulen geht es um das partizipative Entwickeln didaktischer Konzepte sowie das Gestalten von Handlungsmöglichkeiten und -räumen. Ferner geht es um eine Analyse gesellschaftlicher Prozesse und eigener Weltanschauungen, da die digital vernetzte Welt und die daraus resultierenden Phänomene Teil des Metaprozesses (Krotz 2007, 11) sind. Digitalisierung ist ein Produkt menschlicher Kultur (Rat für Kulturelle Bildung 2019) und hat Auswirkungen darauf, wie Menschen leben, welche Wahrnehmungsstrukturen sie ausbilden und nutzen und wie sie ihre Umwelt gestalten. Unterrichtsentwicklung stellt dabei eine kokonstruktive, partizipativ organisierte Gestaltungsaufgabe dar, um etwas Neues in bestehende Strukturen einzubringen und diese zu verändern - insbesondere mit Blick auf den Einsatz digitaler Medien in der Schule (Irion 2016; Irion 2018; Döbeli Honegger 2016). Als grundlegende Kompetenzziele im Rahmen des Seminars «Chancen und Herausforderungen von Schul- und Unterrichtsentwicklung - Digitale Formate im Unterricht an der Grundschule» wurden unter Bezugnahme auf das Modulhandbuch für den Studiengang Master Lehramt Grundschule an der Pädagogischen Hochschule Schwäbisch Gmünd festgelegt:

1. Die Studierenden verfügen über vertiefte Kenntnisse theoretischer Grundlagen in einem ausgewählten Themenbereich.

2. Die Studierenden kennen Methoden und Modelle grundschulbezogener Schul- und Unterrichtsentwicklung; können diese einordnen und reflektiert nutzen.

3. Die Studierenden verfügen über grundlegende Kompetenzen zur Anwendung ausgewählter Modelle - theoretischer und praktischer Natur - in exemplarischen Themenbereichen. 
Das nachfolgende Praxisbeispiel basiert auf Datenmaterial aus Videound Fotoaufzeichnungen sowie aus Forschungstagebüchern.

\subsection{Praxisbeispiel Escape-Room für das Schulmuseum in Schwäbisch Gmünd}

Bei einem Escape-Room geht es darum, Aufgaben und Rätsel zu lösen sowie bestimmten Situationen zu entkommen. Das Spiel basiert auf einer (in den Kleingruppen gemeinsam) festgelegten Storyline. Hierbei kann es sich beispielsweise um eine Reise in die Vergangenheit oder in die Zukunft der Stadtgeschichte oder eine Kriminalgeschichte usw. handeln, die sich auf ausgewählte Themen bezieht. Mit Blick auf den anzubahnenden Kompetenzerwerb, erstellten Studierende Escape Rooms für das Schulmuseum in Schwäbisch Gmünd. Die Spiele wurden im Anschluss der Öffentlichkeit zur Verfügung gestellt.

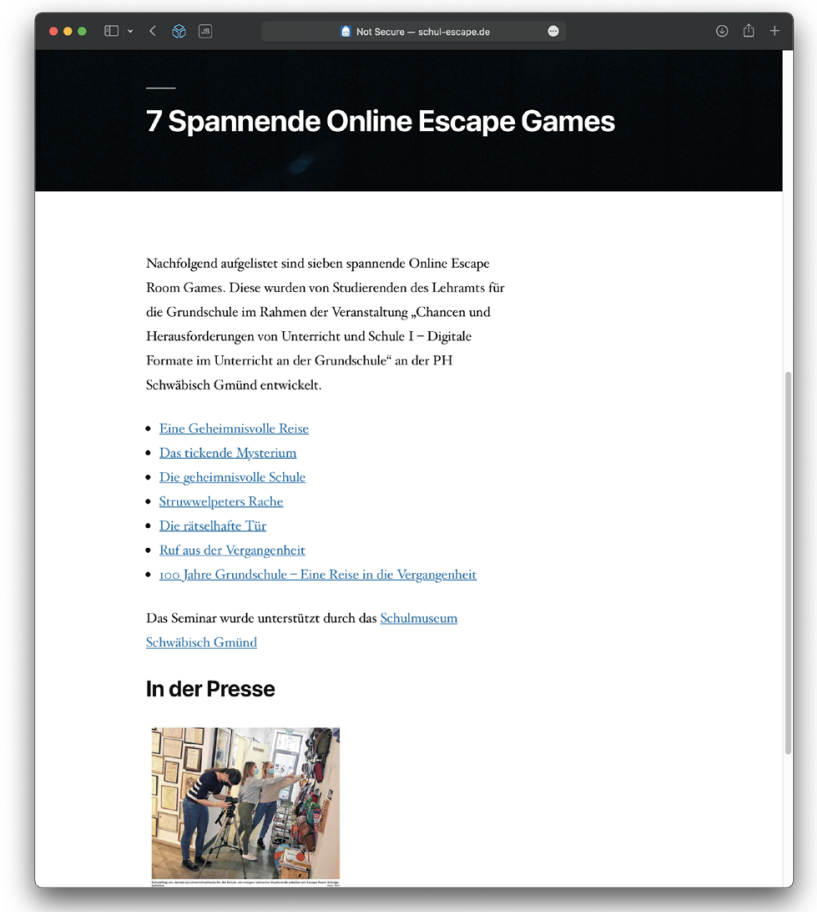

Abb. 3: Escape Games auf www.schul-escape.de. 


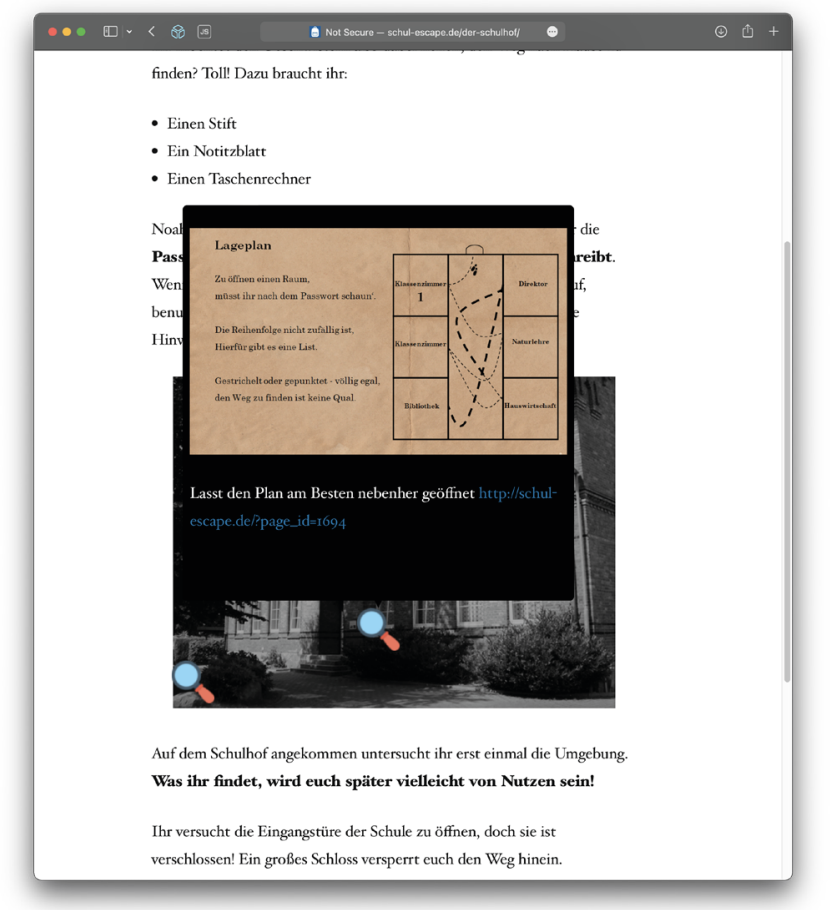

Abb. 4: Einblick in ein Escape Game auf www.schul-escape.de.

Ziel des Kooperationsprojekts war es, dass Studierende das Museum eigenständig erkunden, Schwerpunkte festlegen sowie darauf bezogen eine Storyline für einen Online-Escape Room gestalten und im Anschluss umsetzen. Hierzu erhielten die Gruppen eine übergeordnete Aufgabenstellung sowie zuvor festgelegte Bearbeitungskriterien, die ausreichend Raum für freies und kreatives Schaffen ermöglichten. Auf diese Weise wurde das Projekt eigenständig, partizipativ und kollaborativ geplant, umgesetzt sowie reflektiert, im Sinne einer kritisch-konstruktivistisch orientierten Didaktik. Unterstützung und interdisziplinäre Einblicke lieferten externe Expertinnen und Experten und Coaches. Digitale Endgeräte wurden eingesetzt, um Inhalte, Gegenstände und Räume im Sinne des forschend-entdeckenden Lernens eigenständig zu ergründen. 


\subsection{Projekt- und Seminarablauf}

Aufgrund der im Wintersemester 2020/21 noch vorherrschenden Pandemie-Lage wurde das projektbegleitende Seminar über Zoom durchgeführt. Die online-basierte Organisation des Seminars liess ein Aufbrechen von räumlichen und zeitlichen Strukturen zu: Nicht alle Sitzungen wurden im wöchentlichen Rhythmus durchgeführt, sondern Aufgaben konnten zum Teil auch individuell nach Absprache in den jeweiligen Kleingruppen bearbeitet werden. Das Seminar basierte auf theoretischen und praktischen Einheiten (siehe Abb. 3).
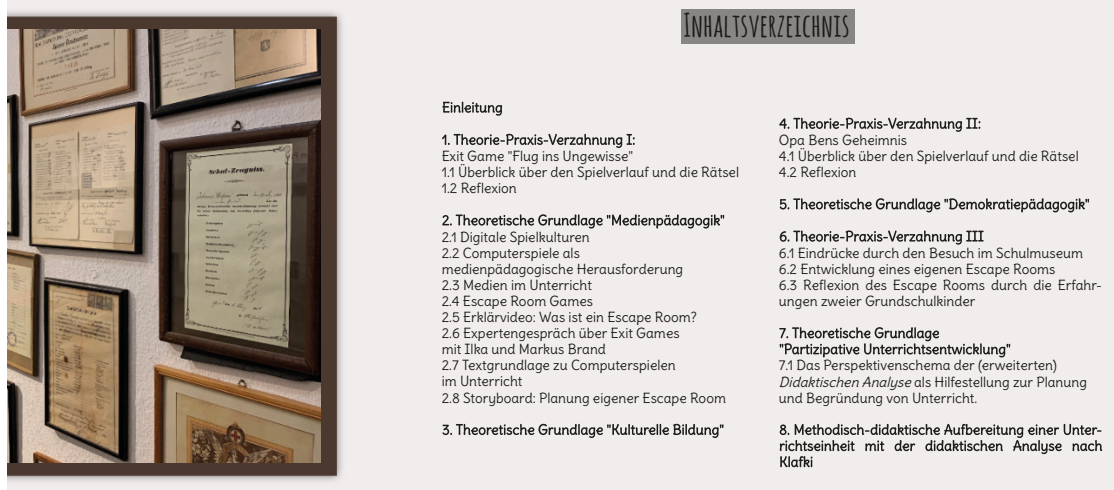

Abb. 5: Seminarstruktur - Auszug aus dem Forschungstagebuch einer Studentin.

Im Rahmen von insgesamt vier Theorie-Sitzungen setzten sich die Studierenden mit Basis-Texten über Medienbildung und -didaktik in der Grundschule und Game-based Learning sowie einem Modell zur Schulentwicklung nach Rolff (2016) und der Ladder of Participation nach Hart (1992) auseinander. Um die Texte zu bearbeiten erhielten die Studierenden Checklisten mit Aufgaben. Um eine sowohl individuelle als auch gruppenbasierte Auseinandersetzung zu gewährleisten, wurden auch sogenannte Break-out Sessions durchgeführt. Jeweils im wöchentlichen Wechsel zu den Theorieeinheiten fanden Workshops statt, sodass sich die Studierenden in die unterschiedlichen digitalen Formate (u. a. Wordpress, bookcreator, Miro-Board storyline, online Rätselformate generieren) einarbeiten konnten. Das Workshop-Format ermöglichte, hierarchische Strukturen 
aufzubrechen, da die Studierenden nach kurzen Einführungsrunden, sich eigenständig über das Miro-Board mit den jeweiligen Formaten beschäftigten. Die Lehrenden wurden zu ihren Begleitern und standen für Rückfragen zur Verfügung.

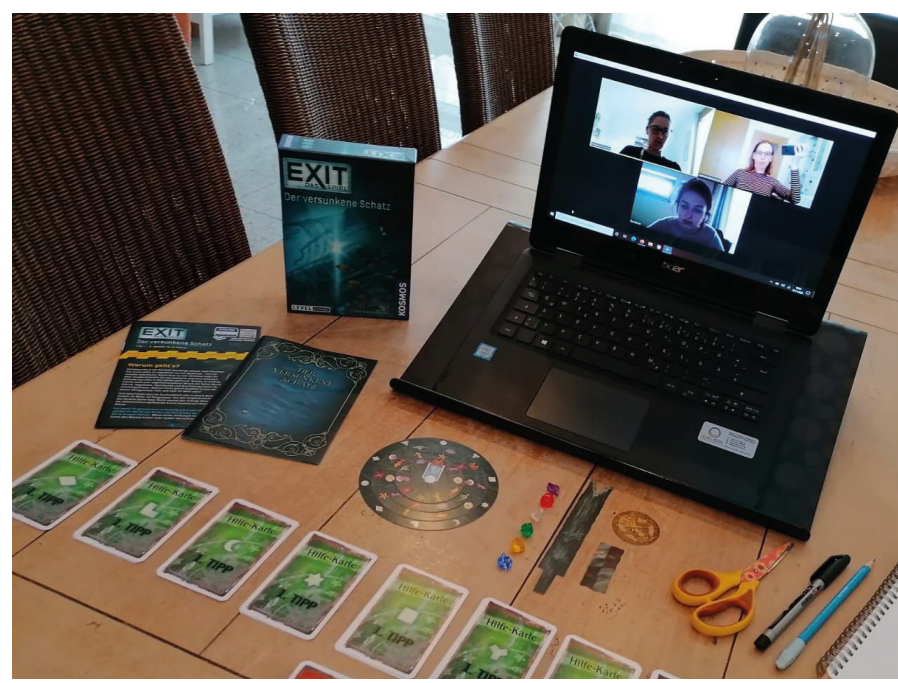

Abb. 6: Individuell gestaltete Gruppenarbeit aus dem Forschungstagebuch einer Studentin.

\subsection{Eindriucke durch den Besuch des SCHULMUSEUMS}

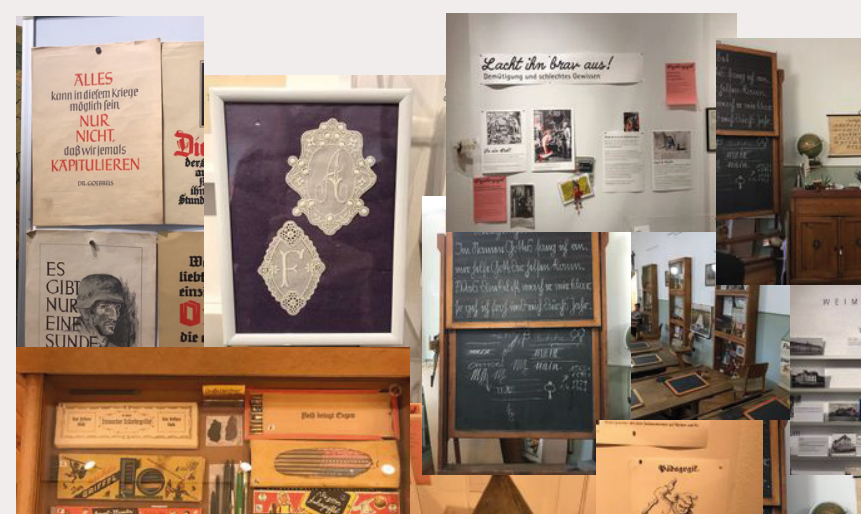

Abb. 7: Eindrücke aus dem Schulmuseum aus dem Forschungstagebuch einer Studentin. 
Nach Abschluss der theoretisch-praktisch angelegten Einheiten besuchten die Kleingruppen das Schulmuseum in Schwäbisch Gmünd, um sich Informationen zu beschaffen und Schwerpunkte für die Storyline zu setzen. Für die Gestaltung des Escape-Rooms standen schliesslich vier Sitzungen à 90 Minuten inklusive Vor- und Nachbereitungszeit zur freien Verfügung, während die Lehrenden auch hier wieder als beratende Begleiterinnen und Begleiter bei Bedarf fungierten. Abschluss bildeten schliesslich zwei weitere Sitzungen für das Spielen und Reflektieren sowie das abschliessende Überarbeiten der Escape-Rooms.

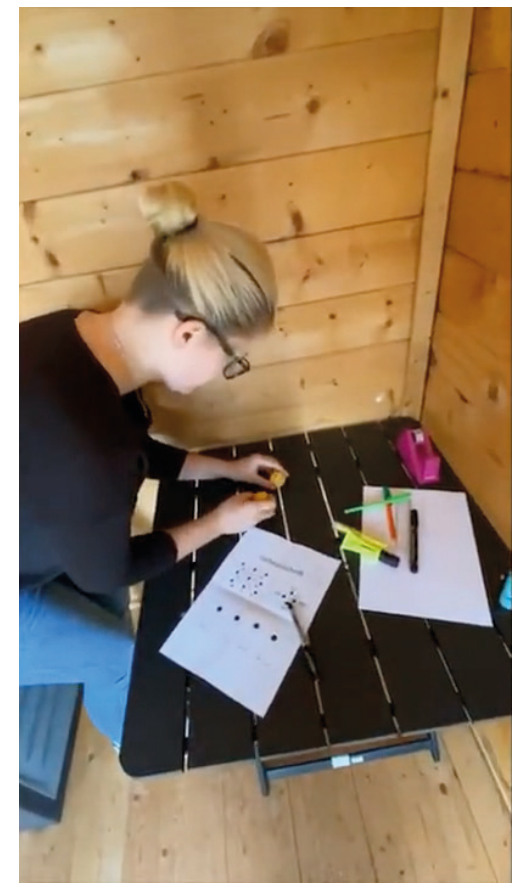

Abb. 8: Ausschnitt aus einem von einer Studierendengruppe erstellten Erklärvideo zum Thema «Was ist ein Escape Room?».

In dem so aufgespannten theoretisch-praktischen Rahmen erarbeiteten sich die Studierenden grundschulpädagogisches Grundwissen, lernten, dieses kritisch zu hinterfragen und anhand des eigenen Modells (Escape-Room) zu reflektieren, um sich durch die Verbindung von Berufsfeldund Wissenschaftsorientierung ( $\Rightarrow$ Unterrichtseinheit) eine Grundlage 
für die eigene theoriegeleitete Praxis zu schaffen. Ziel war (a) der Erwerb spezifischen Wissens über Schulentwicklungsprozesse am Modell, um am Fachdiskurs über die eigene Profession teilnehmen zu können, sowie (b) der Erwerb von Kompetenzen mit Blick auf die eigene Professionalität als Lehrerin/Lehrer. Die offen gehaltene, digitale sowie theoretisch-praktische Durchführung des Seminars sollte dabei insbesondere die Fähigkeit fördern, Bildungsprozesse unter dem Aspekt individueller Voraussetzungen und der selbstständigen Gestaltung von Lehr- und Lernprozessen zu betrachten sowie zu initiieren. Modulabschluss bildete die Ausarbeitung einer methodisch-didaktisch aufbereiteten Unterrichtsreihe für eine 3./4. Grundschulklasse. Dies erfolgte vor dem Hintergrund der aktuellen Pandemie-Lage: Da Museen aufgrund des Lockdowns geschlossen waren, galt es, die Inhalte online der Öffentlichkeit sowie insbesondere Schülerinnen und Schülern zugänglich zu machen.

\section{Fazit}

Vor dem Hintergrund der bisherigen Ausführungen werden die Zusammenhänge zwischen den einzelnen Institutionen mit Fokus auf eine Verschränkung von Theorie und Praxis im Lehramtsstudium sichtbar, die in eine politisch-kulturelle Medienbildung münden könnten (Niesyto 2020; siehe auch Autenrieth und Nickel 2021). Mit Blick auf aktuelle und künftige Herausforderungen in Unterricht, Schule und Gesellschaft sowie mit dem Ziel, dass die Kultur der Digitalität auch eine Kultur der Partizipation darstellt und die Digitalisierung in all ihren Facetten wahrgenommen und gestaltet werden kann, entsteht durch die Zusammenarbeit verschiedener (auch professionspolitischer) Felder der Pädagogik eine Chance, den Metaprozess Digitalisierung subjektorientiert, partizipativ und sozial wie ebenso ethisch verantwortungsvoll mitzugestalten (Autenrieth und Nickel 2020).

Basierend auf den dargelegten Perspektiven ergeben sich für uns daher die folgenden Schlussfolgerungen: Schulen, insbesondere Grundschulen brauchen kompetent ausgebildetes Personal sowie darauf bezogen eine wissenschaftlich fundierte, praxisnahe Aus- und Weiterbildung von Lehrerinnen und Lehrern. Hierfür müssen entsprechende Strukturen 
bereitgestellt werden, um eine (grund-)schulgerechte Medienbildung bzw. Grundbildung mit Medien zu gewährleisten. Situative Handlungspraxis ermöglicht eben diese Professionalisierung durch Kompetenzauf- und -ausbau in der Lehrpersonenaus- und -weiterbildung. Insofern erscheint uns eine dialogisch angelegte, partizipativ organisierte Praxisforschung zwischen Schulen, Hochschulen, Universitäten und örtlich ansässigen Einrichtungen elementar.

Wie aufgezeigt, verstehen wir Studierende sowie Schülerinnen und Schüler dabei als Subjekte und aktive Gestaltende ihres eigenen Lernprozesses. Durch die Umsetzung forschungsbasierter, dialogisch angelegter Projekte direkt an der Schnittstelle von Medien-, Demokratie- und Kultureller Bildung können Lehrende, Studierende sowie Schülerinnen und Schüler partizipativ an der Entwicklung von Schule und Unterricht arbeiten, indem sie Handlungsspielräume erkennen und nutzen. Aus diesem Grund ordnen wir die beschriebene Projektumsetzung im Sinne der Ladder of Participation nach Roger Hart (vgl. 1992) auf der sechsten Stufe des Partizipationsgrades ein. Mit anderen Worten: Um Modul-Strukturen gerecht zu werden, werden Entscheidungen und Projekte von den Lehrenden in der Schule, Hochschule sowie im und mit dem ausserschulischen Lernort initiiert. Studierende, Schülerinnen und Schüler werden demgegenüber informiert und in Abstimmungsprozesse einbezogen. Handlungsmacht, im Sinne Giddens' (vgl. 1988) bedeutet daher hier, dass die beteiligten Akteurinnen und Akteure ihre Fähigkeiten, Ideen und Potenziale über pädagogische Freiheit und durch kreatives Handeln sowie divergentes Denken im Projektprozess entfalten und einbringen können, wenn sie dies möchten. «Handeln hängt von der Fähigkeit des Individuums ab, <einen Unterschied herzustellen〉 zu einem vorher existierenden Zustand oder Ereignisablauf, d. h. irgendeine Form von Macht auszuüben» (Giddens 1988, 66). So können Strukturen, wie eingangs beschrieben, hinterfragt und - bei Bedarf - verändert werden. 


\section{Literatur}

Arnold, Rolf, und Michael Schön. 2019. Ermöglichungsdidaktik: ein Lernbuch. Bern: hep.

Aufenanger, Stefan, Birgit Eickelmann, und Bardo Herzig. 2014. «Medienbildung entlang der Bildungskette. Ein Rahmenkonzept für eine subjektorientierte Förderung von Medienkompetenz im Bildungsverlauf von Kindern und Jugendlichen». Deutsche Telekom Stiftung. https://www.telekom-stiftung.de/ sites/default/files/files/media/publications/buch_medienbildung.bildungskette_end.pdf.

Autenrieth, Daniel, Claudia Baumbusch, und Anja Marquardt. 2020. «Lehren und Lernen mit und über Medien in Kooperation von Schule, Hochschule und Museen: Am Beispiel des Projekts 〈Reuchlin digital»». MedienPädagogik: Zeitschrift für Theorie und Praxis der Medienbildung 17 (Jahrbuch Medienpädagogik): 531-63. https://doi.org/10.21240/mpaed/jbl7/2020.05.21.X.

Autenrieth, Daniel, und Stefanie Nickel. 2020. «Kultur der Digitalität = Kultur der Partizipation?». Medienimpulse 58 (4): 32. https://doi.org/10.21243/MI-04-20-13.

Autenrieth, Daniel, und Stefanie Nickel. 2021. «Politisch-kulturelle Medienbildung. Herausforderungen für Gesellschaft, Schule und Unterricht des 21. Jahrhunderts». Lehren und Lernen. Zeitschrift für Schule und Innovation BadenWürttemberg 47 (3) 3: 6-9.

Beck, John C., und Mitchell Wade. 2004. Got game: how the gamer generation is reshaping business forever. Boston: Harvard Business School Press.

Beranek, Angelika, und Sebastian Ring. 2016. «Nicht nur Spiel - Medienhandeln in digitalen Spielwelten als Vorstufe zu Partizipation». merzWissenschaft Digitale Spiele. https://www.jff.de/veroeffentlichungen/detail/2016-06-digitalespiele/.

Bourdieu, Pierre. 1987. Sozialer Sinn: Kritik der theoretischen Vernunft. Frankfurt am Main: Suhrkamp.

Csikszentmihalyi, Mihaly. 2015. Flow: das Geheimnis des Glücks., 17. Aufl., Stuttgart: Klett-Cotta.

Deci, Edward, und Richard Ryan. 1993. «Die Selbstbestimmungstheorie der Motivation und ihre Bedeutung für die Pädagogik». Zeitschrift für Pädagogik 39: 223-38. https://doi.org/10.25656/01:11173.

Döbeli Honegger, Beat. 2016. Mehr als 0 und 1: Schule in einer digitalisierten Welt. Bern: hep. http://mehralsoundı.ch/Digital/Inhaltsverzeichnis.

Dolata, Ulrich. 2014. «Märkte und Macht der Internetkonzerne: Konzentration - Konkurrenz - Innovationsstrategien». Stuttgarter Beiträge zur Organisations- und Innovationsforschung 2014-04. https://www.sowi.uni-stuttgart.de/ dokumente/forschung/soi/soi_2014_4_Dolata_Maerkte_und_Macht_der_Internetkonzerne.pdf. 
Feierabend, Sabine, Thomas Rathgeb, und Theresa Reutter. 2020. «JIM-Studie 2020. Jugend, Information, Medien. Basisuntersuchung zum Medienumgang 12- bis 19-Jähriger». Herausgegeben von Medienpädagogischer Forschungsverbund Südwest (mpfs). Medienpädagogischer Forschungsverbund Südwest (LFK, LMK). https://www.mpfs.de/fileadmin/files/Studien/JIM/2020/JIM-Studie-2020_Web_final.pdf.

Giddens, Anthony. 1988. Die Konstitution der Gesellschaft. Grundzüge einer Theorie der Strukturierung. Frankfurt/M. und New York: Campus.

Hart, Roger. 1992. «Children's Participation. From Tokenism to Citizenship». Innocenti Essays 4. https://www.unicef-irc.org/publications/100-childrens-participation-from-tokenism-to-citizenship.html.

Helsper, Werner. 2012. «Antinomien im Lehrerhandeln. Professionelle Antinomien - vermeidbare Verstrickung oder pädagogische Notwendigkeit?», Lernende Schule 60.

Huizinga, Johan. 1956. Homo Ludens. Vom Ursprung der Kultur im Spiel. Reinbek: Rowohlt.

Husserl, Edmund. 1996. Die Krise der europäischen Wissenschaften und die transzendentale Phänomenologie, herausgegeben von Elisabeth Ströker, 3. Aufl. Hamburg: Meiner.

Hüther, Gerald, und Christoph Quarch. 2016. Rettet das Spiel! weil Leben mehr als Funktionieren ist. München: Carl Hanser.

Irion, Thomas. 2016. «Digitale Medienbildung in der Grundschule - Primarstufenspezifische und medienpädagogische Anforderungen». In Neue Medien in der Grundschule 2.o. Grundlagen - Konzepte - Perspektiven, herausgegeben von Markus Peschel und Thomas Irion, 16-32. Frankfurt/Main: Grundschulverband. https://doi.org/10.25656/01:16558.

Irion, Thomas. 2018. «Wozu digitale Medien in der Grundschule? Sollte das Thema Digitalisierung in Grundschulen tabuisiert werden?». Grundschule aktuell 142. https://doi.org/10.25656/01:15574.

Koller, Hans-Christoph. 2012. Bildung anders denken: Einführung in die Theorie transformatorischer Bildungsprozesse. Pädagogik. Stuttgart: W. Kohlhammer.

Krempl, Stefan. 2020. «Corona-App per PEPP-PT: Kanzleramt soll bei Apple Druck machen». heise online (blog). 23. April 2020. https://www.heise.de/newsticker/ meldung/Corona-App-per-PEPP-PT-Kanzleramt-soll-bei-Apple-Druck-machen-4708759.html.

Krotz, Friedrich. 2007. Mediatisierung: Fallstudien zum Wandel von Kommunikation, 1. Aufl. Medien, Kultur, Kommunikation., Wiesbaden: VS Verlag für Sozialwissenschaften. https://doi.org/10.1007/978-3-531-90414-6.

Luhmann, Niklas. 1981. Soziologische Aufklärung 3: Soziales System, Gesellschaft, Organisation. https://doi.org/10.1007/978-3-663-01340-2.

McGonigal, Jane. 2012. Reality Is Broken: Why Games Make Us Better and How They Can Change the World [Includes Practical Advice for Gamers]. London: Vintage Books. 
Nickel, Stefanie. 2016. «Demokratie-Lernen - normatives Ideal oder konstruktive Möglichkeit? Eine Untersuchung handlungsleitender Denkstrukturen schulischer Akteur*innen über Demokratie im Kontext Schule und allgemein». Dissertation, Universität Duisburg-Essen, Fakultät für Bildungswissenschaften. https://nbn-resolving.org/urn:nbn:de:hbz:464-20170203-152251-3.

Niesyto, Horst. 2019a. "Ergebnisse des Entwicklungsprojekts dileg-SL. Kernpunkte in teilprojektübergreifender Perspektive». In Digitale Medien in der Grundschullehrerbildung Erfahrungen aus dem Projekt dileg-SL, herausgegeben von Horst Niesyto und Thorsten Junge, München: kopaed.

Niesyto, Horst. 2019b. «Medienpädagogik und digitaler Kapitalismus. Für die Stärkung einer gesellschafts- und medienkritischen Perspektive». KULTURELLE BILDUNG ONLINE. https://doi.org/10.25529/92552.313.

Niesyto, Horst. 2020. «Politisch-kulturelle Medienbildung». https://horst-niesyto. de/politisch-kulturelle-medienbildung/.

Oevermann, Ulrich. 1981. «Professionalisierung der Pädagogik. Professionalisierbarkeit pädagogischen Handelns». Mitschrift eines Vortrags im Sommersemester 1981 an der FU Berlin.

Rat für Kulturelle Bildung, Hrsg. 2019. Jugend/Youtube/Kulturelle Bildung - Horizont 2019 Studie: eine repräsentative Umfrage unter 12- bis 19-jährigen zur Nutzung kultureller Bildungsangebote an digitalen Kulturorten. Essen: Rat für Kulturelle Bildung e. V. https://www.rat-kulturelle-bildung.de/fileadmin/user_upload/pdf/Studie_YouTube_Webversion_final.pdf.

Reich, Kersten. 2002. Konstruktivistische Didaktik: Lehren und Lernen aus interaktionistischer Sicht. Pädagogik und Konstruktivismus., Neuwied: Luchterhand.

Rolff, Hans-Günter. 2016. Schulentwicklung kompakt: Modelle, Instrumente, Perspektiven. 3. vollst. überarb. Auflage, Pädagogik, Weinheim und Basel: Beltz.

Schmidt, Robin. 2020. «Post-digitale Bildung». In Was macht die Digitalisierung mit den Hochschulen?, herausgegeben von Marko Demantowsky, Gerhard Lauer, Robin Schmidt, und Bert te Wildt, 57-70. De Gruyter Oldenbourg. https:// doi.org/10.1515/9783110673265-005.

Simon, Nina. 2010. The Participatory Museum. Santa Cruz, California: Museum 2.0.

Soßdorf, Anna. 2016. Zwischen Like-Button und Parteibuch: die Rolle des Internets in der politischen Partizipation Jugendlicher. Research, Wiesbaden: Springer VS. https://doi.org/10.1007/978-3-658-13932-2.

Stalder, Felix. 2019. Kultur der Digitalität. Edition Suhrkamp. Berlin: Suhrkamp.

Theunert, Helga. 2009. «Medienkompetenz». In Grundbegriffe Medienpädagogik. Praxis, herausgegeben von Bernd Schorb, Günther Anfang, und Kathrin Demmler. München: kopaed.

Trilling, Bernie, und Charles Fadel. 2009. 21st Century Skills. Learning for Life in Our Times. San Francisco: Jossey-Bass/Wiley. 